University of Nebraska - Lincoln

DigitalCommons@University of Nebraska - Lincoln

\title{
Monoclinic Optical Constants, Birefringence, and Dichroism of Slanted Titanium Nanocolumns Determined by Generalized Ellipsometry
}

\author{
Daniel Schmidt \\ University of Nebraska - Lincoln \\ Benjamin Booso \\ Electro-Optics Graduate Program, University of Dayton, Dayton, Ohio \\ Tino Hofmann \\ University of Nebraska - Lincoln, thofmann4@unl.edu \\ Eva Schubert \\ University of Nebraska - Lincoln, efranke3@unl.edu \\ Andrew Sarangan \\ Electro-Optics Graduate Program, University of Dayton, Dayton, Ohio \\ See next page for additional authors \\ Follow this and additional works at: https://digitalcommons.unl.edu/cmrafacpub \\ Part of the Nanoscience and Nanotechnology Commons
}

Schmidt, Daniel; Booso, Benjamin; Hofmann, Tino; Schubert, Eva; Sarangan, Andrew; and Schubert, Mathias, "Monoclinic Optical Constants, Birefringence, and Dichroism of Slanted Titanium Nanocolumns Determined by Generalized Ellipsometry" (2009). Faculty Publications from Nebraska Center for Materials and Nanoscience. 87.

https://digitalcommons.unl.edu/cmrafacpub/87

This Article is brought to you for free and open access by the Materials and Nanoscience, Nebraska Center for (NCMN) at DigitalCommons@University of Nebraska - Lincoln. It has been accepted for inclusion in Faculty Publications from Nebraska Center for Materials and Nanoscience by an authorized administrator of DigitalCommons@University of Nebraska - Lincoln. 


\section{Authors}

Daniel Schmidt, Benjamin Booso, Tino Hofmann, Eva Schubert, Andrew Sarangan, and Mathias Schubert 


\title{
Monoclinic optical constants, birefringence, and dichroism of slanted titanium nanocolumns determined by generalized ellipsometry
}

\author{
Daniel Schmidt, ${ }^{1, a)}$ Benjamin Booso, ${ }^{2}$ Tino Hofmann, ${ }^{1}$ Eva Schubert, ${ }^{1}$ Andrew Sarangan, ${ }^{2}$ \\ and Mathias Schubert ${ }^{1, b)}$ \\ ${ }^{1}$ Department of Electrical Engineering and Nebraska Center for Materials and Nanoscience, University of \\ Nebraska-Lincoln, Lincoln, Nebraska 68588-0511, USA \\ ${ }^{2}$ Electro-Optics Graduate Program, University of Dayton, Dayton, Ohio 45469-0245, USA
}

(Received 10 November 2008; accepted 11 December 2008; published online 8 January 2009)

\begin{abstract}
Generalized spectroscopic ellipsometry determines the principal monoclinic optical constants of thin films consisting of slanted titanium nanocolumns deposited by glancing angle deposition under $85^{\circ}$ incidence and tilted from the surface normal by $47^{\circ}$. Form birefringence measured for wavelengths from 500 to $1000 \mathrm{~nm}$ renders the Ti nanocolumns monoclinic absorbing crystals with $\mathbf{c}$-axis along the nanocolumns, $\mathbf{b}$-axis parallel to the film interface, and $67.5^{\circ}$ monoclinic angle between the $\mathbf{a}$ and c-axes. The columnar thin film reveals anomalous optical dispersion, extreme birefringence, strong dichroism, and differs completely from bulk titanium. Characteristic bulk interband transitions are absent in the spectral range investigated. (c) 2009 American Institute of Physics. [DOI: $10.1063 / 1.3062996]$
\end{abstract}

Slanted metal nanocolumn thin films can be produced by evaporation and tilting the substrate with respect to the direction of the incoming vapor. ${ }^{1}$ The physical properties of such thin films differ drastically from their bulk material. ${ }^{2}$ Intriguing mechanical, magnetic, and optical properties, for example, can be obtained and may be explored in future applications. Form-induced polarization current confinement, crosscoupling between individual nanoelements, and quantization effects will lead to entirely different optical properties unknown from their bulk counterpart. Hodgkinson and $\mathrm{Wu}^{1}$ predicted, and partially determined, strong nanostructure form-induced optical orthorhombic birefringence in slanted nanostructure thin films, also termed columnar thin film (CTF). Deposition conditions such as the angle of the incoming vapor have strong influence on shape and arrangement of the nanostructures. ${ }^{3}$ Controlled CTF growth together with accurate measurement of their anisotropic optical properties will allow for tailoring materials at the nanometer scale to achieve desired optical applications such as omnidirectional mirrors and thin film polarization filters. In this letter we report accurate and complete determination of the intrinsic optical properties of slanted metal nanocolumn thin films. We extend the prediction of Hodgkinson and $\mathrm{Wu}^{1}$ by demonstrating that CTFs can also possess monoclinic properties. Monoclinic and triclinic thin film optical properties can be determined by generalized ellipsometry (GE). This concept, developed for spectroscopic applications, ${ }^{4-6}$ was demonstrated for orthorhombic ${ }^{7,8}$ and triclinic ${ }^{9}$ thin film situations recently. Beydaghyan et al. ${ }^{8}$ reported on GE measurements of slanted silicon oxide nanoneedles deposited under various deposition angles. Analysis of the ellipsometry data was done under the assumption of homogeneous thin film orthorhombic biaxial properties and validity of the effective medium concept. The authors calculated depolarization factors for directions perpendicular to the $\mathbf{c}$-axis and reported porosity parameters for different deposition angles from their op-

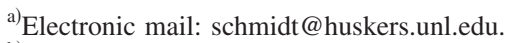

${ }^{b)}$ Electronic mail: schubert@engr.unl.edu.
}

tical model analysis. The Euler angle $\theta$ describing the inclination of the homogeneous orthorhombic biaxial thin film $c$-direction was provided and found in reasonable comparison with scanning electron microscopy images.

GE allows for determination of complete and accurate sets of optical constants for biaxial (dielectrically anisotropic) materials. For arbitrarily anisotropic materials, the complex ratio $\rho$ of the $s$ - and $p$-polarized reflectivities depends on the polarization state of the incident light. Measurement of $\rho$ can be addressed within different presentations of the electromagnetic plane wave response. Here we make use of the Stokes description including Mueller matrices. Realvalued Mueller matrix elements $M_{i j}$ connect the Stokes parameters before and after sample interaction. ${ }^{10}$

The Stokes vector elements for the traditional $p$-s polarization system are $S_{0}=I_{p}+I_{s}, S_{1}=I_{p}-I_{s}, S_{2}=I_{45}-I_{-45}$, and $S_{3}=I_{\sigma+}-I_{\sigma-}$, where $I_{p}, I_{s}, I_{45}, I_{-45}, I_{\sigma+}$, and $I_{\sigma-}$ denote the intensities for the $p-, s-,+45^{\circ},-45^{\circ}$, right-, and left-handed circularly polarized light components, respectively. Data analysis requires nonlinear regression methods, where measured and calculated GE data are matched as close as possible by varying appropriate physical model parameters. The calculations require setup of models for geometry, layer structure, and polarizability properties of materials involved in the sample of interest. ${ }^{4-6}$ Due to the complexity of this subject, thorough discussion of this issue is beyond the scope of this paper, and referral is made to the literature. ${ }^{4-7,9-12}$ The linear polarizability response of CTFs due to an electric field $\mathbf{E}$ is a superposition of contributions along certain (major) intrinsic directions ("unit-cell vectors") $\mathbf{a}=a_{x} \mathbf{x}+a_{y} \mathbf{y}$ $+a_{z} \mathbf{z}, \quad \mathbf{b}=b_{x} \mathbf{x}+b_{y} \mathbf{y}+b_{z} \mathbf{z}, \quad \mathbf{c}=c_{x} \mathbf{x}+c_{y} \mathbf{y}+c_{z} \mathbf{z}: \quad \mathbf{P}=\varrho_{a} \mathbf{a}+\varrho_{\mathbf{b}} \mathbf{b}$ $+\varrho_{\mathbf{c}}$ c. In the laboratory Cartesian coordinates, the CTF is described by the second rank polarizability tensor $\chi$ and $\mathbf{P}$ $=\chi \mathbf{E}$.

The laboratory Cartesian coordinate frame $(x, y, z)$ is defined by the plane of incidence $(x, z)$ and the sample surface $(x, y)$. This Cartesian frame is rotated by the Euler angles $\varphi, \psi, \theta$ to an auxiliary system $(\xi, \eta, \zeta)$, with $\zeta$ being parallel to $\mathbf{c .}^{10,11}$ For orthorhombic, tetragonal, hexagonal, and trigo- 


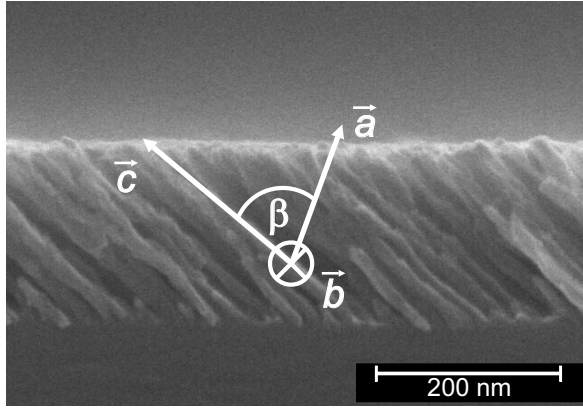

FIG. 1. SEM micrograph of slanted Ti nanocolumns grown on a Si substrate. The overlaid coordinate system indicates the orientation of the monoclinic biaxial system with its internal c-axis along the nanocolumns and $\mathbf{b}$-axis parallel to the film interface. The angle $\beta$ between axes a and $\mathbf{c}$ is $67.5^{\circ}$.

nal systems, $\varphi, \psi, \theta$ exist with $\chi$ being diagonal in $(\xi, \eta, \zeta)$. For monoclinic and triclinic systems an additional projection operation $\mathbf{T}$ onto the orthogonal auxiliary system $(\xi, \eta, \zeta)$ is necessary, $^{13}$

$$
T=\left(\begin{array}{ccc}
\sin \beta & \frac{\cos \gamma-\cos \beta \cos \alpha}{\sin \beta} & 0 \\
0 & \sqrt{\sin ^{2} \alpha-\frac{\cos \gamma-\cos \beta \cos \alpha}{\sin \beta}} & 0 \\
\cos \beta & \cos \alpha & 1
\end{array}\right) .
$$

Additional parameters $\alpha, \beta, \gamma$ are introduced into the analysis procedure, which differentiate between orthorhombic $\left(\alpha=\beta=\gamma=90^{\circ}\right)$, monoclinic $\left(\beta \neq 90^{\circ}\right)$, or triclinic $(\alpha \neq \beta \neq \gamma)$ biaxial optical properties. As a result, one obtains functions $\varrho_{a}, \varrho_{b}, \varrho_{c}$, Euler angles $\varphi, \psi, \theta$, internal angles $\alpha, \beta, \gamma$, and thin film thickness $d$. Note that angle and thickness parameters are not allowed to vary with wavelength. The major optical constants $\left(n_{j}+\sqrt{-1} k_{j}\right) \equiv \sqrt{\left(1+\varrho_{j}\right)}$ $(j=a, b, c)$ can thus be extracted on a point-by-point basis, i.e., without any physical line shape implementations.

Titanium nanocolumns were deposited by electron-beam glancing angle deposition in high vacuum onto silicon substrates. The $\mathrm{Si}$ substrates had a native oxide layer of $\approx 3 \mathrm{~nm}$. A commercially available (Torr International, Inc.) electronbeam system was utilized. The distance between source and the customized sample stage is $500 \mathrm{~mm}$. The sample was tilted by $85^{\circ}$ with respect to the incident particle flux direction. The constant evaporation rate of $\approx 5 \AA / \mathrm{s}$ was monitored with a quartz crystal microbalance deposition controller. Figure 1 depicts a high-resolution scanning electron microscope (SEM) image of a sample edge view. Angle-resolved (angle of incidence $\Phi_{a}$ and sample rotation angle $\varphi$ ) spectroscopic Mueller matrix ellipsometry measurements were performed using a commercial instrument (M2000, J. A. Woollam Co., Inc.) within a spectral range from $\lambda=500$ to $1000 \mathrm{~nm}$. The ellipsometer was mounted on an automatic variable $\Phi_{a}$ and sample rotator $\varphi$ stage. $\Phi_{a}$ was varied from $45^{\circ}$ to $75^{\circ}$ in steps of $10^{\circ}$, while $\varphi$ was varied from $0^{\circ}$ to $360^{\circ}$ in steps of $5^{\circ}$. The polarizer-compensator-sample-analyzer ellipsometer is capable of measuring 11 out of 16 Mueller matrix elements normalized to $M_{11}$ (except for elements in the fourth row). ${ }^{10}$

Figure 2 depicts exemplary GE data at $\lambda=850 \mathrm{~nm}$. Model and experimental data are in perfect agreement, also

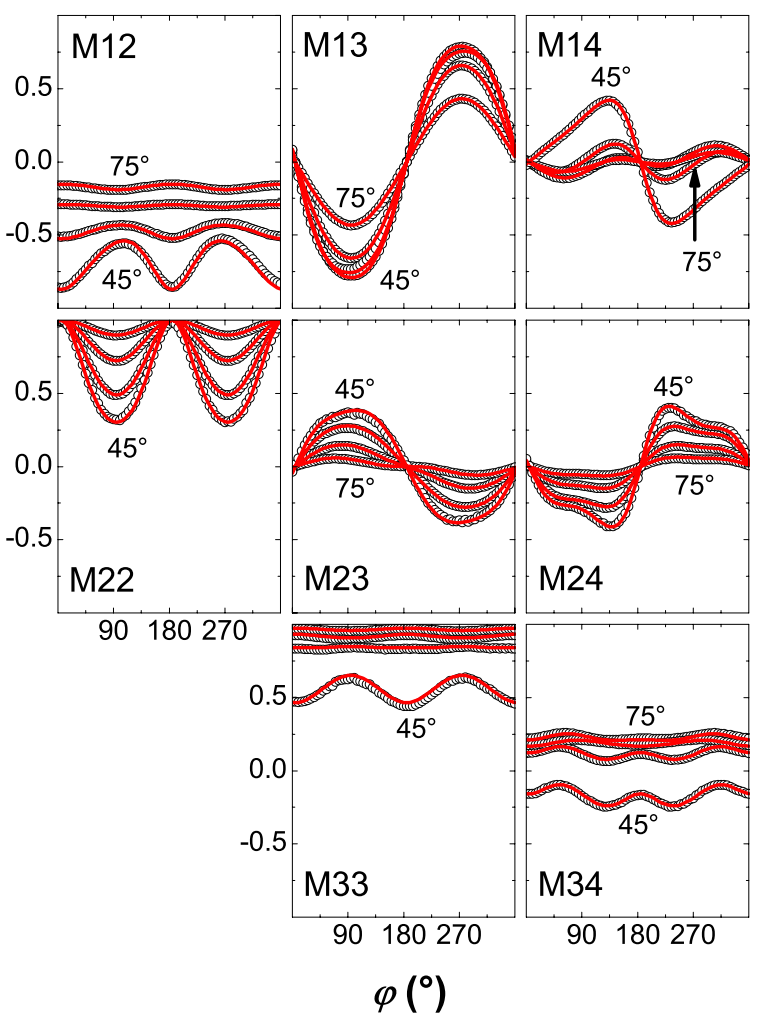

FIG. 2. (Color online) Exemplary experimental (symbols) and best-match (solid lines) calculated GE data vs sample azimuth $\varphi$ and angle of incidence $\Phi_{a}$ at $\lambda=850 \mathrm{~nm}$.

for all other wavelengths measured in the investigated spectral region. In Fig. 2 the highly anisotropic nature of the Ti CTF becomes obvious. Note that there is no repetition of data over one full rotation except for symmetry with respect to pseudoisotropic $\varphi$ positions, and data over one full turn should be measured in order to fully evaluate the optical properties. For brevity, we have only plotted nonredundant elements of the Mueller matrix, and the remaining elements can be obtained by symmetry as described previously. ${ }^{12}$ Two pseudoisotropic sample orientations can be identified in Fig. 2 at $\varphi \approx 5^{\circ}$ and $\varphi \approx 185^{\circ}$, which occur when the plane of incidence is parallel to the slanting direction of the nanocolumns. The best-match geometrical properties for our Ti CTF sample reveal a thin film thickness of $178.9 \pm 0.1 \mathrm{~nm}$, which is in very good agreement with the thickness obtained from the SEM in Fig. $1(196 \mathrm{~nm})$. The difference can be well explained by variation across the sample and uncertainties in SEM image analysis. The structural inclination of the slanted nanocolumns of $47^{\circ}$ is in excellent agreement with the Euler angle $\theta=46.7^{\circ} \pm 0.3^{\circ}$. We further obtained $\varphi$ $=85.25^{\circ} \pm 0.01^{\circ}, \beta=67.5^{\circ} \pm 0.4^{\circ}$, and $\psi=0^{\circ}, \gamma=\alpha=90^{\circ}$. Thus, the Ti CTF possesses monoclinic optical properties as indicated in Fig. 1. The monoclinic angle can be understood by considering polarization charge transfer along the nanocolumns. The columns are able to exchange charges at the bottom of the thin film due to their conducting nucleation layer but are isolated at the top from each other. Therefore the effective overall dipole moment for electric fields perpendicular to the columns and within the slanting plane is tilted toward the surface normal. Accordingly, the monoclinic angle should change with the slanting angle and converge to $90^{\circ}$ with increasing thickness. 


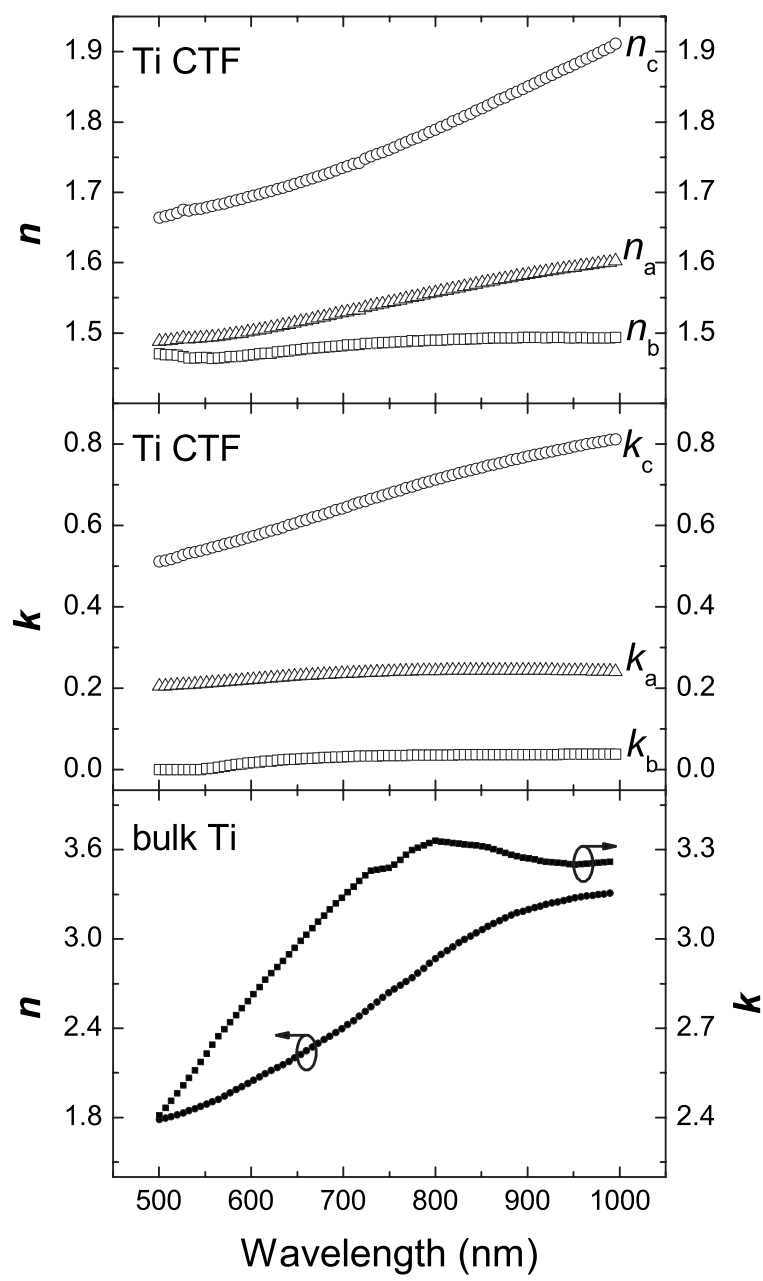

FIG. 3. Monoclinic optical constants $n_{a}, n_{b}, n_{c}$ and $k_{a}, k_{b}, k_{c}$ obtained from the best-model calculation for Ti CTF sample in Fig. 1, and bulk Ti optical constants for comparison.

Figure 3 depicts wavelength dependencies of refractive indices $n_{j}$ and absorption coefficients $k_{j}(j=a, b, c)$. The index of refraction $n_{c}$ and absorption coefficient $k_{c}$ are largest (along the nanocolumn $\mathbf{c}$-axis). Very strong birefringence can be seen between all polarizabilities, which also reveals anomalous dispersion. There is no crossover between optical constants. Note that almost no absorption occurs parallel to the film-substrate interface over the entire investigated spectral range. It is also interesting to note that there is very small wavelength dispersion for all refractive indices. Hodgkinson and $\mathrm{Wu}^{\mathrm{l}}$ reported earlier that the three principal refractive indices in a similar biaxial orthorhombic medium satisfy $n_{c}$ $>n_{a}>n_{b}$, which is in agreement with our result here. The strong dichroism follows the same order $\left(k_{c}>k_{a}>k_{b}\right)$ as $n$. All Ti CTF optical constants differ significantly from bulk $n$ and $k$ of polycrystalline $\mathrm{Ti}^{14}{ }^{14}$ shown also in Fig. 3. Particularly noticeable is the absence of the characteristic bulk Ti interband transitions at around $\lambda=750 \mathrm{~nm}$. Comparison with results for Si nanocolumns reported by Beydaghyan et al. ${ }^{8}$ and Hsu et al. ${ }^{15}$ are of interest. Both groups applied effective medium approximations for rendering a biaxial inhomogeneous material matching their measured GE data with model calculations. However, lack of model details makes direct comparison difficult. In particular, the polarizability functions along the assumed orthorhombic axes were not reported. As pointed out by Aspnes, ${ }^{16}$ effective medium approximations are not applicable to CTFs because the length of the columns is not negligible against the wavelength. Augmentation of the simplest form of effective medium approximations results in linear approximations averaging void fraction of index unity with fraction of nanocolumns, and which is estimated at $70 \%$ here. This correlation would rescale the $y$-axis of the CTF in Fig. 3 accordingly. However, the resulting $n$ and $k$ spectra do not reflect those of one individual nanocolumn, which should be of cylindrical symmetry. Instead, it is the response of all nanocolumns and their specific arrangement with respect to each other, which produce the $(n, k)$ spectra and monoclinic properties valid for the entire thin film. Description of anisotropic functions $\varrho_{a}, \varrho_{b}, \varrho_{c}$ in terms of porosity requires revised effective medium concepts; instead, the only matter of fact extractable from (generalized) ellipsometry measurements are complex-valued functions $\varrho_{a}, \varrho_{b}, \varrho_{c}$ pertinent to intrinsic axes $\mathbf{a}, \mathbf{b}, \mathbf{c}$, their (internal) angles $\alpha, \beta, \gamma$, and external (Cartesian Euler) angles $\varphi, \theta, \psi$. Those may then be compared and evaluated in terms of nanostructure geometry and arrangement. We propose GE as useful technique for optical characterization of complex, highly anisotropic nanostructure thin films.

The authors acknowledge financial support from Ohio Department of Development, NSF in MRSEC QSPIN, CoE, UNL, and J.A. Woollam Foundation as well as fruitful discussions with Qiwen Zhan.

${ }^{1}$ I. J. Hodgkinson and Q. H. Wu, Birefringent Thin Films and Polarizing Elements (World Scientific, Singapore, 1998).

${ }^{2}$ M. M. Hawkeye and M. J. Brett, J. Vac. Sci. Technol. A 25, 1317 (2007).

${ }^{3}$ C. Buzea, K. Kaminska, G. Beydaghyan, T. Brown, C. Elliott, C. Dean, and K. Robbie, J. Vac. Sci. Technol. B 23, 2545 (2005).

${ }^{4}$ M. Schubert, Phys. Rev. B 53, 4265 (1996).

${ }^{5}$ M. Schubert, Thin Solid Films 313-314, 323 (1998).

${ }^{6}$ M. Schubert, Ann. Phys. 15, 480 (2006).

${ }^{7}$ M. Schubert and W. Dollase, Opt. Lett. 27, 2073 (2002).

${ }^{8}$ G. Beydaghyan, C. Buzea, Y. Chi, C. Elliott, and K. Robbie, Appl. Phys. Lett. 87, 153103 (2005).

${ }^{9}$ M. Dressel, B. Gompf, D. Faltermeier, A. K. Tripathi, J. Pflaum, and M. Schubert, Opt. Express 16, 19770 (2008).

${ }^{10}$ Handbook of Ellipsometry, edited by H. G. Tompkins and E. A. Irene (Springer, Heidelberg, 2004).

${ }^{11}$ M. Schubert, Infrared Ellipsometry on Semiconductor Layer Structures (Springer, Heidelberg, 2004).

${ }^{12}$ D. Schmidt, E. Schubert, and M. Schubert, Phys. Status Solidi A 205, 748 (2008).

${ }^{13}$ I.-H. Suh, Y.-S. Park, and J.-G. Kim, J. Appl. Crystallogr. 33, 994 (2000).

${ }^{14}$ Handbook of Optical Constants of Solids, edited by E. D. Palik (Academic, Boston, 1991).

${ }^{15}$ S.-H. Hsu, E.-S. Liu, Y.-C. Chang, J. N. Hilfiker, Y. D. Kim, T. J. Kim, C.-J. Lin, and G.-R. Lin, Phys. Status Solidi A 205, 876 (2008).

${ }^{16}$ D. E. Aspnes, Thin Solid Films 89, 249 (1982). 